\title{
IMAGE REGISTRATION USING ADVANCED TOPOLOGY PRESERVING RELAXATION LABELING
}

\author{
Chan-IL Kim, Ji-Ae Park, Su Yang, Yoon-Nyun Kim, Hee-Jun Park, \\ and Jong-Ha Lee* \\ ${ }^{1}$ Department of Biomedical Engineering, Keimyung University, Daegu, Korea \\ segeberg@kmu. ac. kr
}

\begin{abstract}
This paper presents a relaxation labeling technique with newly defined compatibility measures for solving a general non-rigid point matching problem. In the literature, there exists a point matching method using relaxation labeling, however, the compatibility coefficients always take a binary value zero or one depending on whether a point and a neighboring point have corresponding points. Our approach generalizes this relaxation labeling approach. The compatibility coefficients take n-discrete values which measures the correlation between edges. We use log-polar diagram to compute correlations. Through simulations, we show that this topology preserving relaxation method improves the matching performance significantly compared to other state-of-the-art algorithms such as shape context, thin plate spline-robust point matching, robust point matching by preserving local neighborhood structures and coherent point drift.
\end{abstract}

\section{KEYWORDS}

Point pattern matching, graph matching, registration, relaxation labelling

\section{INTRODUCTION}

The point matching is widely used in computer vision and pattern recognition because point representations are generally easy to extract [1],[2]. Point matching can be categorized as rigid matching and non-rigid matching based on the deformation of objects captured in the images [3]. Compare with the rigid case, non-rigid matching is more complicated. Generally, there are two unknown operations: correspondence and transformation. Most non-rigid point matching approaches use an iterated estimation framework to find appropriate correspondence and transformation [4]. The Iterated Closest Point (ICP) algorithm is one of the most well known heuristic approaches [5]. It utilizes the relationship by assigning the correspondence with binary values zero and one. However, this binary assumption is no longer valid in the case of non-rigid transformation, especially when the deformations are large. The Thin Plate Spline Robust Point Matching (TPS-RPM) algorithm is an Expectation Maximization (EM) algorithm to jointly solve for the feature correspondence as well as the geometric transformation [6]. The cost function that is being minimized is the sum of Euclidean distances between points. In the TPS-RPM, the binary correspondence value of the ICP is relaxed to the continuous value between zero and one. This soft-assign method improves the matching performance because the correspondences are able to improve gradually and continuously without jumping around in the space of binary permutation

Natarajan Meghanathan et al. (Eds) : ACSIT, SIPM, CMIT, CoNeCo - 2016

pp. 31-42, 2016. (C) CS \& IT-CSCP 2016

DOI : $10.5121 / \mathrm{csit} .2016 .60804$ 
matrices [7]. The algorithm is robust compared to the ICP in the non-rigid case, but the joint estimation of correspondences and transformation increases complexity. In addition, the Euclidean distance makes sense only when there are at least rough initial alignments of the shapes. If the initialization is not aligned well, the matching result is poor. Recently, the Shape Context (SC) algorithm has been proposed [8]. It is an object recognizer based on the shape. For each point, the distribution of the distance and orientation are estimated to the neighboring points through a histogram. This distribution is used as the attribute relations for the points. The correspondences can be decided by comparing each point's attributes in one set with the attributes of the other. Because only the attributes are compared, the searching for the correspondences can be conducted more easily compared to the ICP and the TPS-RPM. Generally, the SC performs better in handling complex patterns than the TPS-RPM. Another interesting approach of point matching is a kernel correlation-based point matching [9]. The cost function is proportional to the correlation of two kernel density estimates. The work was extended by using the L2 distance between mixtures of Gaussian representing the point set data in [10]. The Coherent Point Drift (CPD) algorithm is another probabilistic algorithm [11]. They proposed to use the displacement filed between the point sets following the motion coherence theory. They also successfully extend the general non-rigid registration framework, and show that TPS-RPM is its special case. The Robust Point Matching by preserving Local Neighborhood Structures (RPM-LNS) introduces the notion of a neighborhood structure for the general point matching problem [9]. The RPM-LNS uses a relaxation labeling method with binary value coefficient. This approach is based on the assumption that although the absolute distance between two points may change significantly under non-rigid deformation, the neighborhood structure of a point is generally well preserved. The cost function is formulated as an optimization problem to preserve local neighborhood relations.

The relaxation labeling technique is an iterative procedure that reduces local ambiguities and achieves global consistency by exploiting contextual information which is quantitatively represented by "compatibility coefficients" [10], [11]. In this paper, we define optimal compatibility coefficients for a relaxation labeling to solve a non-rigid point matching problem. Our work is inspired by that of [9]. There, point matching problem is formulated to preserve local neighborhood structure during matching process and a relaxation labeling technique is applied to provide optimal solutions. The present work, however, differs from that of [9] in the determination of compatibility coefficients. In [9], the compatibility coefficient values were only considered as binary value "zero" or "one" depending on whether a point and its neighboring point have corresponding points. In this paper, the correlation between points and neighbors is measured by a compatibility function and represents as " $n$ " discrete values. In order to do this, we quantify the amount of smoothness and similarity between points and neighbors using a log-polar diagram. Consider a set of vectors originating from a point to all adjacent points. We compute distance and angle from an origin point to the rest of points by counting the number of bins. This contextual information combined with relaxation labeling process is used to search for an optimized correspondence. After finding the correspondence using several relaxation labeling updates, TPS is used to find the optimal transformation [12]. These two processes are iterated until the optimal correspondence and transformation are found [13]. The proposed relaxation labeling with compatibility function preserves topology, thus we call our method the Topology Preserving Relaxation Labeling (TPRL). The simulation results show that TPRL is stable and robust under all deformations, noises, outliers, rotations, and occlusions.

The reminder of the paper is organized as follows. We review the relaxation labeling problem formulation as an optimization problem in Section II. In Section III, we define new compatibility coefficients and present a relaxation labeling process for point matching problem. We then show the robustness of our approach compared to the other algorithms in Section IV. Finally, Section V discusses the conclusions of the paper. 


\section{PROBLEM FORMULATION}

Let be a set of control points in a model set and be a set of control points in the target set. In a matching problem, one-to-one matching is desired, but in general, one-to-one matching is not possible because of outliers. To handle this problem, two point sets are augmented to and by introducing a dummy or a nil point. Then common points can be matched one-to-one and outliers can be matched to a dummy point. Every point has its adjacent points called neighbors. For a given point, one can select adjacent points, is the total number of adjacent points of. Similarly, for a point, adjacent points are and is the total number of adjacent points of .

In this paper, the matching problem is formulated as the graph edge matching problem [5]. Each point is a node of a graph, and a point and its adjacent points make edges of a graph. Then the problem is to maximize the number of matched edges between two graphs. For this purpose, we determine the fuzzy correspondence matrix. Each entry of has continuous value between $[0,1]$ that indicates the weight of the correspondence between and .Then the optimal match is found by maximizing the energy function as follows.

$$
\hat{P}=\arg \max _{P} C(\hat{S}, \hat{T}, P)
$$

where

$$
C(\hat{S}, \hat{T}, P)=\sum_{i=1}^{I} \sum_{x=1}^{X} \sum_{j=1}^{J} \sum_{y=1}^{Y} P_{s_{i} t_{j}} P_{a_{x}^{s_{i}} a_{y}^{t_{j}}}
$$

The above equations are subject to the following constraints $\sum_{j=1}^{J+1} P_{s_{i} t_{j}}=1$ for $i=1,2, \ldots, I$ and $\sum_{i=1}^{I+1} P_{s_{i} t_{j}}=1$ for $j=1,2, \ldots, J$

Defining adjacent points are very important in maximizing the matching probability and reducing the computational complexity. In this paper, an adjacent point searching boundary is assumed for any given point. The searching boundary is defined as a circle with the radius of the median length of all edges. If a point is residing in the circle centered at $s_{i}$, it becomes an adjacent point

$a^{s_{i}}$ of $s_{i}$. The median length of total edges is an efficient metric for determining the search boundary, if points are selected non-uniformly. If points are placed randomly, however, there are both crowded areas and sparse areas. Logically, a point in a crowded area should have more adjacent points and thus more connected edges. A point in a sparse area should have less adjacent points with less connected edges. If we assign same number of adjacent points to each point, a point in a sparse area may be associated with a distant point in a crowded area as the adjacent point. Furthermore, points in a crowded area may not necessarily form edges, and points in a sparse area may form useless edges. This unnecessary assignment leads to increased computational complexity. The proposed adjacent point definition allows the assignment of more connected edges in a crowded area and fewer connected edges in a sparse area. This flexibility reduces the complexity and increases the reliability of the algorithm.

\section{POINT CORRESPONDENCE USING RELAXATION LABELING}

Here, we use a relaxation labeling technique to solve an optimization problem of non-rigid point matching. The relaxation labeling is an iterative process to reduce ambiguities in assigning labels to a set of objects by incorporating the contextual information. The contextual information is 
usually represented as compatibility coefficients. It is widely known that the relaxation labeling process is greatly affected by the choice of the compatibility coefficients [14],[15]. Thus, we propose compatibility coefficients that preserve a topology during the matching processes.

Initially, each point is assigned with a set of matching probability based on the shape context. After the initial probability assignment, the relaxation labeling process updates the matching probability iteratively. The purpose of the subsequent process is to assign matching probability that maximize $C(\hat{S}, \hat{T}, P)$ under the relaxed condition as $P_{s_{i} t_{j}} \in[0,1]$. At the end of the labeling process, it is expected that each point will have one unambiguous matching probability. We follow the relaxation updating rule as follows [16].

1. Compute the compatibility coefficients which impose smoothness and similarity constraints between points.

2. Compute the support function from all compatibility coefficients related to the point.

3. Update the matching probability depending on its support function.

The determination of compatibility coefficients is important because the performance of the relaxation labeling depends on them. In the previous work, the compatibility coefficients are defined as either 0 or 1, a binary value [9]. As a key contribution, we define new compatibility coefficients to relax this binary value into the multiple discrete values. In our approach, the compatibility coefficients quantify the degree of agreement between the hypothesis that $s_{i}$ matches to $t_{j}$ and $a_{x}^{s_{i}}$ matches to $a_{y}^{t_{j}}$. To represent the matching strength between the pair of points and the pair of adjacent points, we use a log-polar diagram. Let the diagram distance and angle originating from $s_{i}$ to $a_{x}^{s_{i}}$ be $d\left(s_{i}, a_{x}^{s_{i}}\right)$ and $l\left(s_{i}, a_{x}^{s_{i}}\right)$, respectively. Every point can be an origin of a shape context and the origin varies with points in consideration to calculate the diagram distance and angle. In the diagram, the distance is defined as 0 in the origin and incremented by 1 towards the outer bins. The bins are uniform in log-polar space, which makes the descriptor more sensitive to positions of adjacent points than to those of points far away. For the geometrical angle calculation, the original shape context is not rotation invariant. To initially align, the mass center of a point set is used as a reference point, and we use the direction from a point to the center of mass as the positive $\mathrm{x}$-axis [9]. From this axis, the geometrical angle is incremented by 1 as the counter clockwise direction. An example is shown in Fig. 1. In this example, all adjacent points of $s_{1}$ and $t_{1}$, which are $a_{1}^{s_{1}}, a_{2}^{s_{2}}, a_{1}^{t_{1}}$ and $a_{2}^{t_{2}}$, have the magnitude of distance of two and the angle of one.

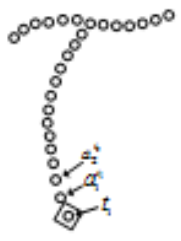

(a)

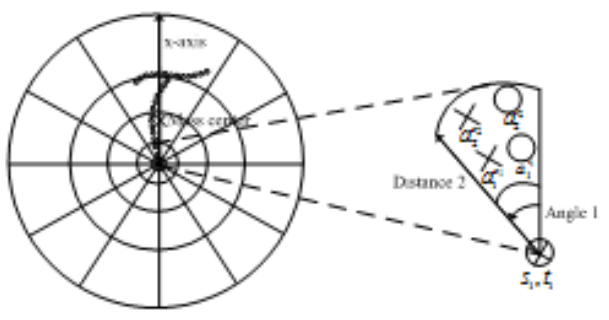

(b)

Fig. 1. Structure based distance and angle computation. (a) Sample points from the deformed shape "T". (b) Example shape context of a reference sample marked by $\diamond$. The log-polar bins diagram is used to compute structural distance and angle. We use five bins for the distance 0 to 4 from the origin and the angle 0 to 12 from the $\mathrm{x}$-axis. 
The compatibility coefficients are represented by the smoothness and the similarity between the pair of points and the pair of adjacent points. In the non-rigid deformation, although the absolute distance between two points may change significantly, the adjacent point structure is generally well preserved. This happens more strongly if the distance between point and its adjacent point is closer. Thus the first constraint of the compatibility coefficients, smoothness, is measured by the diagram distance between $s_{i}$ and $a_{x}^{s_{i}}$ as below.

$$
\alpha\left(s_{i}, a_{x}^{s_{i}}\right)=\left(1-\frac{d_{i}\left(s_{i}, a_{x}^{s_{i}}\right)}{\max \left(d_{i}\left(s_{i}, a_{x}^{s_{i}}\right)\right)}\right)
$$

where $\max \left(d_{i}\left(s_{i}, a_{x}^{s_{i}}\right)\right)$ is the longest edge in the graph. Two points $s_{i}$ and $a_{x}^{s_{i}}$ are the most salient if $\alpha\left(s_{i}, a_{x}^{s_{i}}\right)$ is 1 and the least compatible if $\alpha\left(s_{i}, a_{x}^{s_{i}}\right)$ is 0 . The second constraint, similarity, is measured by the combined difference of structural distance and angle of initial edge $\left(s_{i}, a_{x}^{s_{i}}\right)$ and the final edge $\left(t_{j}, a_{y}^{t_{j}}\right)$. This constraint imposes that if an edge $\left(s_{i}, a_{x}^{s_{i}}\right)$ has less distance and angle differences with the edge $\left(t_{j}, a_{y}^{t_{j}}\right)$, then it has higher probability of matching. The disparity between edges $\left(s_{i}, a_{x}^{s_{i}}\right)$ and $\left(t_{j}, a_{y}^{t_{j}}\right)$ is defined as follows.

$$
\begin{aligned}
& \beta\left(s_{i}, a_{x}^{s_{i}} ; t_{j}, a_{y}^{t_{j}}\right)=\left(1-\left|\frac{d_{i}\left(s_{i}, a_{x}^{s_{i}}\right)-d_{j}\left(t_{j}, a_{y}^{t_{j}}\right)}{\max \left\{d_{i}\left(s_{i}, a_{x}^{s_{i}}\right)-d_{j}\left(t_{j}, a_{y}^{t_{j}}\right)\right\}}\right|\right) \\
& \gamma\left(s_{i}, a_{x}^{s_{i}} ; t_{j}, a_{y}^{t_{j}}\right)=\left(1-\left|\frac{l_{i}\left(s_{i}, a_{x}^{s_{i}}\right)-l_{j}\left(t_{j}, a_{y}^{t_{j}}\right)}{\max \left\{l_{i}\left(s_{i}, a_{x}^{s_{i}}\right)-l_{j}\left(t_{j}, a_{y}^{t_{j}}\right)\right\}}\right|\right)
\end{aligned}
$$

If two edges $\left(s_{i}, a_{x}^{s_{i}}\right)$ and $\left(t_{j}, a_{y}^{t_{j}}\right)$ have same geometrical distance and angle, then they are the most compatible. We define the total compatibility coefficient by

$$
r_{s_{i} t_{j}}\left(a_{x}^{s_{i}} a_{y}^{t_{j}}\right)=\alpha\left(s_{i}, a_{x}^{s_{i}}\right) \cdot \beta\left(s_{i}, a_{x}^{s_{i}} ; t_{j}, a_{y}^{t_{j}}\right) \cdot \gamma\left(s_{i}, a_{x}^{s_{i}} ; t_{j}, a_{y}^{t_{j}}\right)
$$

Clearly, $r_{s_{i} t_{j}}\left(a_{x}^{s_{i}} a_{y}^{t_{j}}\right)$ ranges from 0 to 1 . High value of $r_{s_{i} t_{j}}\left(a_{x}^{s_{i}} a_{y}^{t_{j}}\right)$ corresponds to high matching probability between $\left(s_{i}, a_{x}^{s_{i}}\right)$ and $\left(t_{j}, a_{y}^{t_{j}}\right)$, and the low value corresponds to incompatibility. The support function $q_{s_{i} t_{j}}$ in the k-th iteration is given by

$$
q_{s_{i} t_{j}}^{k}=\sum_{i=1}^{I} \sum_{j=1}^{J} r_{s_{i} t_{j}}\left(a_{x}^{s_{i}} a_{y}^{t_{j}}\right) p_{a_{x}^{s_{s}} a_{y}^{t_{j}}}^{k}=\sum_{i=1}^{I} \alpha\left(s_{i}, a_{x}^{s_{i}}\right) \sum_{j=1}^{J} \beta\left(s_{i}, a_{x}^{s_{i}} ; t_{j}, a_{y}^{t_{j}}\right) \cdot \gamma\left(s_{i}, a_{x}^{s_{i}} ; t_{j}, a_{y}^{t_{j}}\right) p_{a_{x}^{s_{i}} a_{y}^{t_{j}}}^{k}
$$

Note that we use $p_{a_{i}^{m} a_{j}^{k}}^{k}$ to weight $r_{s_{i} t_{j}}\left(a_{x}^{s_{i}} a_{y}^{t_{j}}\right)$ because the support also depends on the likelihood of pair of adjacent points matching probability. Finally, $p_{s_{i} t_{j}}^{k}$ is updated according

$$
p_{s_{i} t_{j}}^{k+1}=p_{s_{i} t_{j}}^{k} q_{s_{i} t_{j}}^{k} / \sum_{j=1}^{J} p_{s_{i} a_{i}^{t_{j}}}^{k} q_{s_{i} a_{i}^{t_{j}}}^{k}
$$

The iterative process can be summarized as follows. If a matching probability between $s_{i}$ and $t_{j}$ is supported from their adjacent points $a_{x}^{s_{i}}$ and $a_{y}^{t_{j}}$, then the chance of being matched increases. The probability decreases if they have relatively less supporting data from their adjacent points. 
After pre-defined iteration, the estimated matching probability is assigned to every point. The points with maximum matching probability less than 0.95 are labeled as outliers by matching them to dummy points. The outlier rejection is performed throughout the optimization process.

The strategy of iterated point correspondence and transformation estimations is widely used for non-rigid point matching [6]. In this work, we use the thin plate spline (TPS) model which is used for representing flexible coordinate transformations. The matched point pairs from several relaxation updates are used to estimate the parameters of TPS deformation model. The estimated parameters are used to transform the model set to bring to close to the target set. Suppose point $\left(x_{i}, y_{i}\right)$ is matched to $\left(u_{i}, v_{i}\right)$ for $i=1,2, \ldots, n$, let $z_{i}=f\left(x_{i}, y_{i}\right)$ be the target function value at location $\left(x_{i}, y_{i}\right)$, we set $z_{i}$ equal to $u_{i}$ and $v_{i}$ in turn to obtain one continuous transformation for each coordinate. The TPS interpolant $f(x, y)$ minimizes the bending energy

$$
I(f)=\iint_{i^{2}}\left(\frac{\partial^{2} f}{\partial x^{2}}\right)^{2}+2\left(\frac{\partial^{2} f}{\partial x \partial y}\right)^{2}+\left(\frac{\partial^{2} f}{\partial y^{2}}\right)^{2} d x d y
$$

And has the solution of the form

$$
f(x, y)=a_{1}+a_{x} x+a_{y} y+\sum_{i=1}^{n} w_{i} U\left(\left\|\left(x_{i}, y_{i}\right)-(x, y)\right\|\right) \text { where } U(r) \text { is the kernel function, }
$$

taking the form of $U(r)=r^{2} \log r^{2}$. If error appears in the matching results, we use regularization to trade off between exact interpolation and minimizing the bending energy as follows.

$$
H[f]=\sum_{i=1}^{n}\left(v_{i}-f\left(x_{i}, y_{i}\right)\right)^{2}+\lambda I_{f}
$$

The regularization parameter $\lambda$ controls the amount of smoothing. Finding correspondence and transformation process is alternatively iterated. Finally, one finds the desired correspondence matrix and the mapping function

\section{VALIDATION, COMPARATIVE ANALYSIS AND PERFORMANCE EVALUATION}

In order to access the performance of the Topology Preserving Relaxation Labeling (TPRL) point matching method, we compare our matching results with state-of-the-art algorithms such as the Shape Context (SC), the Thin Plate Spline-Robust Point Matching (TPS-RPM), the Robust Point Matching by preserving Local Neighborhood Structure (RPM-LNS), and Coherent Point Drift (CPD) method. The Iterated Closest Point (ICP), the correlation-based approach, and the L2 distance approach was not considered in this paper since they have much larger registration errors compared to the algorithms mentioned above

\subsection{Experiments Based on Synthetic Data}

We test algorithms with respect to different degrees of deformation, different amount of noise and outliers, and different rotation. We use two different shape. First model set is totally selected 96 points along the outline of a fish shape, closed contour shape. The second model set uses 108 points points from a Chinese character ("blessing"), an open contour shape. It is a more complex pattern than a fish shape. 
In each test, one of distortions is applied to a model set to create a target set. In the first test, the model set is warped through progressively larger degrees of non-rigid warping. The warped data sets are used as the target set. In the second test, we add certain amount of Gaussian noise to the moderately deformed model set and obtain the new target set. The purpose is to test the algorithms' tolerance to noise. In the third test, different amount of outliers were added to the deformed model sets. Finally, we rotate deformed model sets in a counter clockwise direction for the rotation test. Fig. 2. shows examples of synthesized data sets [6]. A total of 100 samples are generated in each distortion level. The matching performance of each algorithm is compared by mean and standard deviation of RMS distance error over 100 trials in each distortion level.
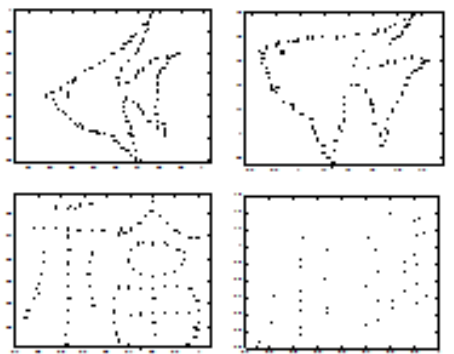

(a) Original
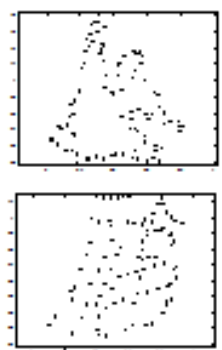

(c) Noise
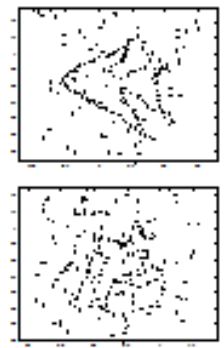

(d) Outlier
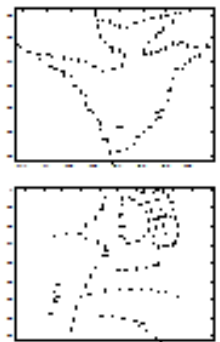

(e) Rotation
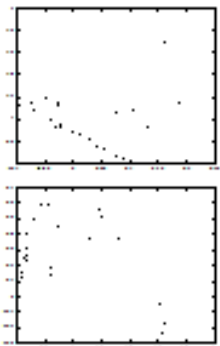

(f) Occlusion

Fig. 2. Synthesized data sets for statistical tests. Model sets are shown in the first column. Column 2-5 shows the target sets for the deformation, noises, outliers, rotations, and occlusion.

We use root mean square (RMS) error for the registration error metric [19]. It is computed as the registration error between the last warped model set and the target set, and is defined by the following equation:

$$
R M S=\sqrt{\frac{1}{M} \sum_{i=1}^{M}\left(s_{i}-t_{i}\right)^{2}}
$$

where $M$ is the total number of points. The statistical matching results (error means and standard deviations for each setting) are shown in Fig. 3.
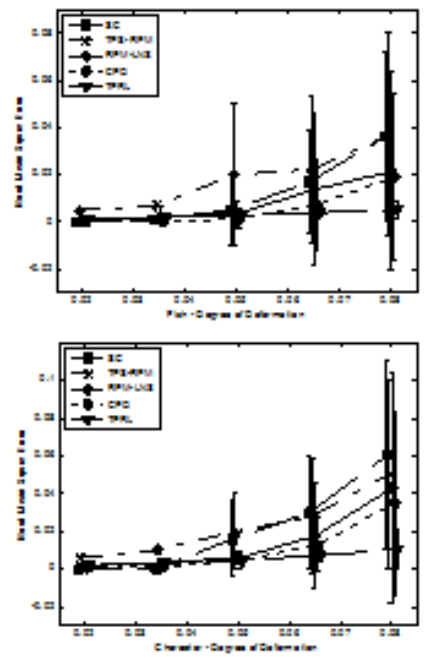

(a) Deformation test
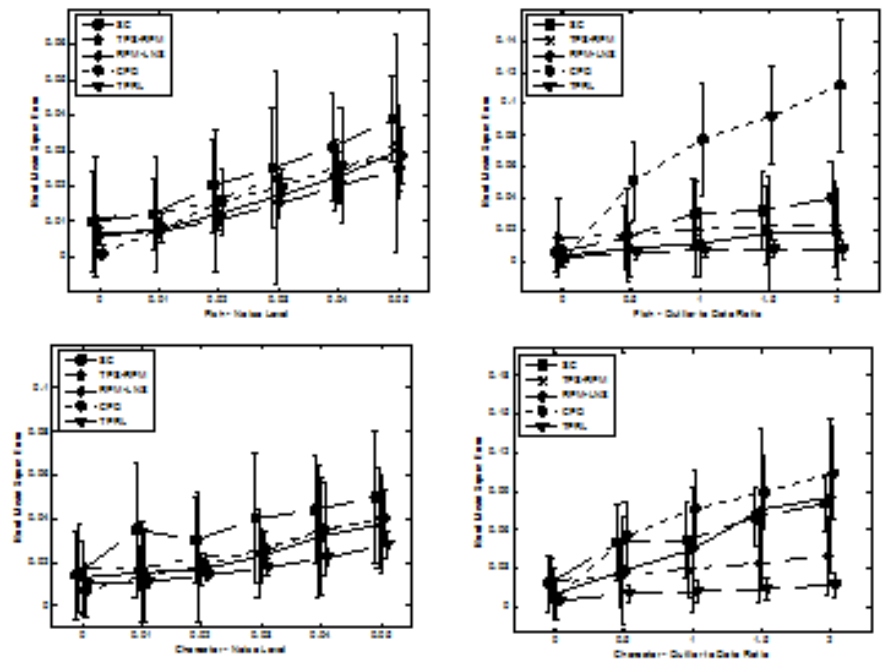

(b) Noise test

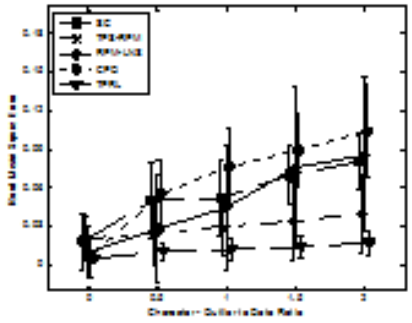

(c) Outlier test 

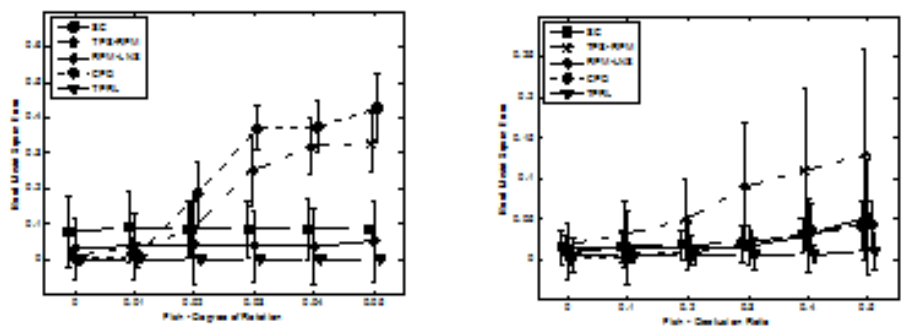

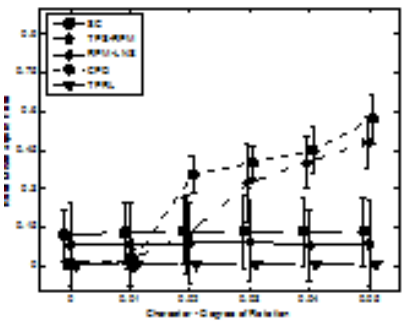

(d) Rotation test

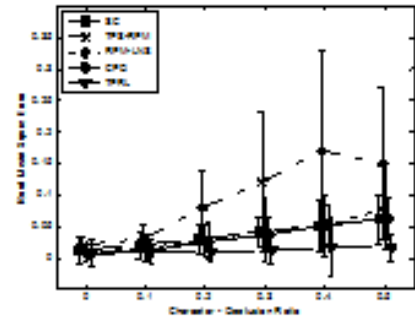

(e) Occlusion test

Fig. 3. Comparison of the matching performance of TPRL ( $\nabla)$ with shape context ( $\square)$, TPS-RPM (*), RPM-LNS $(\diamond)$, and CPD $(O)$ for the fish and character shape, respectively. The error bars indicate the standard deviation of the error over 100 trials.

\subsubsection{Deformation}

In this section, we evaluate algorithm performances under different degree of deformations. Fig. $3 \mathrm{a}$ show the results for the four matching strategies on the fish data set and the character data set. According to the results, TPRL method outperforms the other algorithms, SC, TPS-RPM, RPMLNS, and CPD method. The standard deviation as well as the mean of RMS error is significantly lower than other algorithms. At 0.02 degree of deformation, five algorithms achieve very similar matching performance in both fish and character shape. However, as the degree of deformation increases, we observe that the SC, TPS-RPM, RPM-LNS, and CPD method give poor results while the TPRL method shows the robustness to large deformations. In fact, it seems that the TPRL method is not much affected by deformations. These results show the robustness of the TPRL method under large deformed data sets.

\subsubsection{Noise}

The presence of noise makes the point's location ambiguous. Therefore, this type of data is more challenging than the deformation data. In this section, the performance is measured with a significant amount of noise. Fig. $3 \mathrm{~b}$ presents the results for matching strategies for fish and character shapes. The results show that all algorithms are affected by this type of data distortion. However, we notice that the TPRL method compensates the location ambiguity and finds more accurate correspondences than other three. In contrast, the result of the SC method is the most influenced by noise among four algorithms. The descriptor alone in the SC confuses the real data point and noise since there is no mechanism to distinguish data point and noise in the same descriptor bin. The TPRL method reduces this ambiguity by using topology preserving scheme.

\subsubsection{Outliers}

In addition to the deformation and noise, present outlier further complicates the point matching problem. To evaluate the performance of our method with outliers, we added maximum 405 points outliers in the target set. Fig. 3c displays the RMS error with respect to outlier levels. From 
the result, we can notice that SC in both shapes and TPS-RPM in a character shape are easily confused by outliers and start to fail once the outlier level becomes relatively high. TPS-RPM in the fish shape and RPM-LNS in both shapes seem not much affected by outliers but the error is still higher than TPRL. TPRL is very robust regardless of the outlier level.

\subsubsection{Rotation}

In Fig. 3d, we evaluate the influence of rotation. From the result, we notice that the applied transformation (rotation) does not affect the performance of SC, RPM-LNS and TPRL. All error curves except TPS-RPM are relatively constant at approximately $0.08 \mathrm{~mm}$ for $\mathrm{SC}, 0.03 \mathrm{~mm}$ for RPM-LNS and $0.002 \mathrm{~mm}$ for TPRL in a fish shape; and $0.12 \mathrm{~mm}$ for SC, $0.08 \mathrm{~mm}$ for RPM-LNS and $0.005 \mathrm{~mm}$ for TPRL in the character shape. Note that until 30 degrees of rotation, the error of TPS-RPM is lower than SC and RPM-LNS. But from 90 degree of rotation, TPS-RPM deteriorates quickly and finally reaches $0.33 \pm 0.085 \mathrm{~mm}$ for the fish shape and $0.48 \pm 0.1 \mathrm{~mm}$ for the character shape. TPRL is rotation invariant and consistently outperforms three other algorithms in all degrees of rotations.

\subsubsection{Occlusion}

Occlusion is also important degradation in real applications. We use six occlusion levels to test the five algorithms. As shown in Fig 3e, the performance of the TPS-RPM algorithm deteriorates quickly compared to the other four algorithms. The TPRL algorithm preserves the structure well under the large occlusion ratio and achieves the best result in both fish and character shape synthetic data.

\subsection{Convergence of Relaxation Labeling}

According to the consistency theory, the relaxation labeling process increases the overall consistency by reducing local ambiguities [11],[20]. During the experiments, we verified that the labeling update process converge to an unambiguous solution starting from an arbitrary initialization. Fig. 4 below shows the matching probability of four randomly selected points in 105105 point matching experiments in IV-A. We can observe that while 300 iterations of relaxation labeling updates, the ambiguity of matches decreases and 104 points goes to zero except one point which find their correspondence and goes to one. From the experiments, most elements of matrix do converge to zero to one.

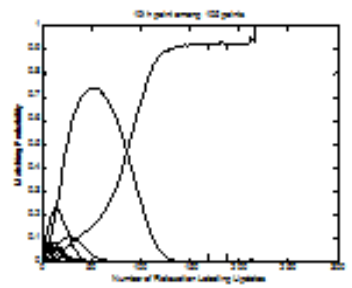

(a)

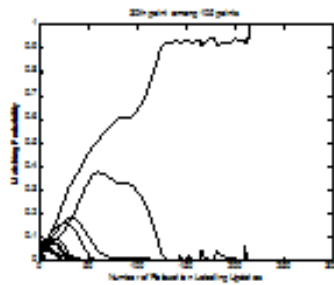

(b)

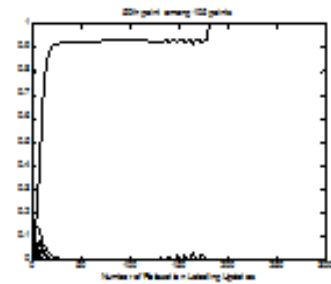

(c)

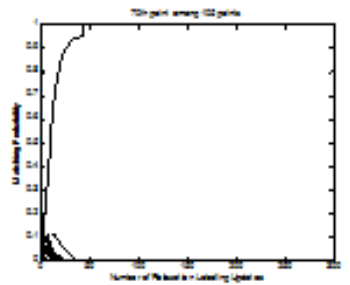

(d)

Fig. 4. The matching probability of (a) 10th, (b) 30th, (c) 50th, (d) 70th points among 105 points in 105 105 matching experiments.

\subsection{Experiments Based on Real World Data Set}

We also conducted experiments on real image data sets. For the real world experiments, we obtained ten frames (10th, 20th, 30th, 40th, 50th, 60th, 70th, 80th, 90th, 100th frame) of Carnegie 
Mellon University hotel sequence as shown in Fig. 4. The database consists of 100 frames of a moving sequence of a toy hotel. Totally 100 points were manually selected in each of the images. Then we conducted four different experiments, 100 against 100, 100 against 90, 100 against 80, and finally 100 against 70 points for every ten image frames defined above.
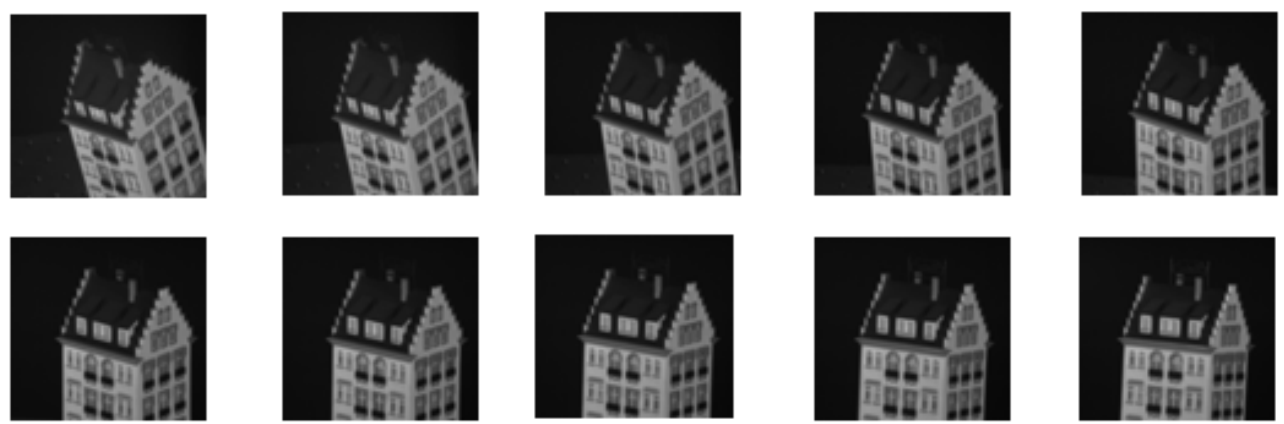

Fig. 4. Sequence images of toy hotel. Frames 10, 20, 30, 40, 50 (top). Frames 60, 70, 80, 90, 100 (bottom).

Fig. 5 shows the results for these experiments. The average RMS distance between each pair and its standard deviation is taken for the statistics.

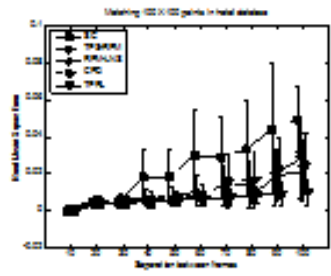

(a)

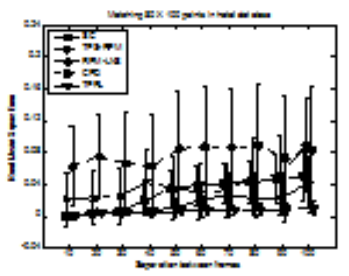

(b)

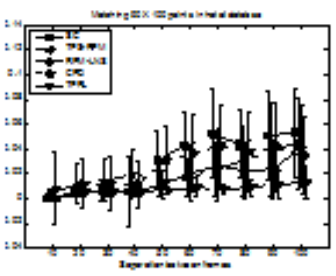

(c)

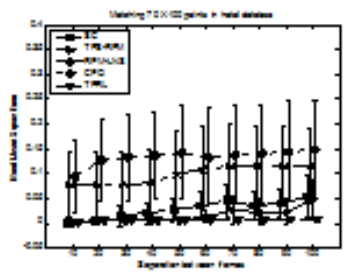

(d)

Fig. 5. Comparison of the matching performance of TPRL $(\nabla)$ with shape context ( $\square)$, TPS-RPM (*), RPM-LNS $(\diamond)$, and CPD $(\mathrm{O})$ in the hotels sequence for increasing frame separation and different target set sizes ((a) 100 100, (b) 90 100, (c) 80 100, (d) 70 100). Error bars correspond to the standard deviation of each pair's RMS error.

In these experiments, we notice that as the relative sizes of the patterns become largely different, the TPRL algorithm performs significantly better than the competing algorithms. The outperformance of the TPRL algorithm is also observable when the target image sequence number is increasing.

\subsection{Experiments Based on Large Data Set}

We also test the TPRL performance under much larger data set. For the model set, 1000 points are extracted from the hey image shown in Fig. 10. To distort the data set, Gaussian random ground function is used. After non-rigid deformation, we have deleted 500 points for one data set and we have added 1000 to 2000 outliers which was generate outliers randomly from a normal zero-mean distribution for other data set. The TPRL performs well under large point sets.

\subsection{Processing Times}

In order to compare algorithms, it is necessary to analyze the complexity of each algorithm. Assume that both model set and target set have $N$ points. The algorithms are based on the NPhard problem and have similar computational complexity of $O\left(N^{2}\right)$ for matching in $\mathbb{R}^{2}$ Among the compared algorithms, the CPD method performs the fastest registration time under large distortion of data set. The TPRL algorithm takes slightly longer time to compute compared 
to CPD algorithm. For 105 points of model set and deformed target set matching, the TPRL takes about 2.1 seconds on a desktop PC with Intel Core 2 Duo @ 2.13GHz and 2GB RAM

\section{CONCLUSIONS}

A novel approach called the TPRL algorithm for the non-rigid point matching problem using a relaxation labelling method with newly defined compatibility coefficients is presented. The matching probability is updated by relaxation process taking into account of the proposed compatibility. A comparative analysis is performed against other algorithms, SC, TPS-RPM, RPM-LNS, and CPD. The TPRL algorithm shows superior performance in both closed (fish) and opens (character) contour examples. We also showed that the TPRL algorithm always obtained the highest matching results compared to other four algorithms under different degree of deformation, noises, outliers, rotation, and occlusion.

\section{ACKNOWLEDGEMENTS}

This work was supported by the Technological Innovation R\&D Program (S2173477) funded by the Small and Medium Business Administration(SMBA, Korea) and this research was supported by Basic Science Research Program through the National Research Foundation of Korea(NRF) funded by the Ministry of Education(2014R1A1A2056420) and this work was supported by the INNOPOLIS Program (16DGI1082).

\section{REFERENCES}

[1] L.G. Brown(1992), "A Survey of Image Registration Techniques," ACM Computing Surveys, vol. 24, no. 4, pp. 325-376.

[2] H. J. Johnson and G. E. Christensen(2002), "Consistent Landmark and Intensity-Based Image Registration,” IEEE Trans. Medical Imaging, vol. 21, no. 5, pp. 450-461.

[3] B. Zitova and J. Flusser(2003), "Image Registration Methods: A Survey," Image and Vision Computing, vol. 21, no 11, pp. 977-1000.

[4] A. Rangarajan, H. Chui, and F. Bookstein(1999), "The Softassign Procrustes Matching Algorithm," Medical Image Analysis, vol. 4, pp. 1-17.

[5] P. J. Besl and N.D. Mckay(1992), “ A Method for Registration of 3-D Shapes,” IEEE Trans. Pattern Analysis and Machine Intelligence, vol. 14, no. 2, pp. 239-256.

[6] H. Chui and A. Rangarajan(2003), "A New Point Matching Algorithm for Non-Rigid Registration," Computer Vision and Image Understanding, vol. 89, no. 23, pp. 114-141.

[7] A. Rangarajan, S. Gold, E. Mjolsness(1996), "A Novel Optimizing Network Architecture with Applications," Neural Computing, vol. 8, no. 5, pp. 1041-1060.

[8] S. Belongie, J. Malik, and J. Puzicha(2002), "Shape Matching and Object Recognition Using Shape Contexts," IEEE Trans. Pattern Analysis and Machine Intelligence, vol. 24, no. 4, pp. 509-522.

[9] Y. Tsin and T. Kanade(2004), "A Correlation-Based Approach to robust Point Set Registration," Proc. 8th European Conf. Computer Vision, pp. 558-569.

[10] B. Jian and B. Vemuri(2005), "A Robust Algorithm for Point Set Registration Using Mixture of Gaussians," Proc. 10th IEEE Int'l Conf. Computer Vision, pp. 1246-1251. 
[11] A. Myronenko, X. Song, and M.A. Carreira-Perpinan(2007), "Non-rigid Point Registration: Coherent Point Drift,” Advances in Neural Information Processing Systems, vol. 19, pp. 1009-1016.

[12] Y. Zheng and D. Doermann(2006), "Robust Point Matching for Nonrigid Shapes by Preserving Local Neighborhood Structures," IEEE Trans. Pattern Analysis and Machine Intelligence, vol. 28, no. 4, pp. 643-649.

[13] A. Rosenfeld, R.A. Hummel, and S.W. Zucker(1976), "Scene Labeling by Relaxation Operations," IEEE Trans. System, Man, and Cybernetics, vol. 6, no. 6, pp. 420-433.

[14] R.A. Hummel and S.W. Zucker(1983), “On the Foundations of Relaxation Labeling Processes,” IEEE Trans. Pattern Analysis and Machine Intelligence, vol. 5, no. 3, pp. 267-287.

[15] F.L. Bookstein(1989), "Principal warps: Thin-plate Splines and the decomposition of deformations," IEEE Trans. Pattern Analysis and Machine Intelligence, vol. 11, no. 6, pp. 567-585.

[16] A.D.J. Cross and E.R. Hancock(1988), "Graph Matching with a Dual-Step EM Algorithm," IEEE Trans. Pattern Analysis and Machine Intelligence, vol. 20, no. 11, pp. 1236-1253.

[17] S. Peleg and A. Rosenfeld(1978), "Determining Compatibility Coefficients for Curve Enhancement Relaxation Processes,” IEEE Trans. System, Man, and Cybermetrics, vol. 8, no. 7, pp. 548-555.

[18] M. Pelillo and M. Refice(1994), "Learning Compatibility Coefficients for Relaxation Labeling Processes,” IEEE Trans. Pattern Analysis and Machine Intelligence, vol. 16, no. 9, pp. 933-945.

[19] Q.X. Wu and D. Pairman(1995), “A Relaxation Labeling Technique for Computing Sea Surface Velocities from Sea Surface Temperature,” IEEE Trans. Geoscience and Remote Sending, vol. 33, no. 1, pp. 216-220.

[20] A.L. Yulle and N.M. Grzywacz(1989), "A Mathematical Analysis of the Motion Coherence Theory," International Journal of Computer Vision, vol. 3, no. 2, pp. 155-175.

[21] K. Mikolajczyk and C. Schmid(2005), “A Performance Evaluation of Local Descriptors," IEEE Trans. Pattern Analysis and Machine Intelligence, vol. 27, no. 10, pp. 1615-1630.

[22] V. Zagrodsky, V. Walimbe, C.R. Castro-Parehja, J.X. Qin, J.M. Song, and R. Shekar(2005), "Registration-assisted Segmentation of Real-time 3-D Echocardiographic Data using Deformable Models,” IEEE Trans. Medical Imaging, vol. 24, no. 9, pp. 1089-1099.

[23] S.W. Zucker, E.V. Krishnamurthy, and R.L. Haar(1978), "Relaxation Processes for Scene Labeling: Convergence, Speed, and Stability,” IEEE Trans. Systems, Man and Cybernetics, vol. 8, no. 1, pp. 41-48. 